\title{
Companies compete over mutation-specific melanoma drugs
}

When a doctor spots a cancerous skin growth, simple surgery is often sufficient to rid the patient of cancer. Once melanoma has spread throughout the body, however, few treatment options exist. Neither chemotherapy nor the immune protein interleukin-2-the two therapies currently approved for treating metastatic melanoma-offer much hope. But a new generation of melanoma drugs nearing approval that target a common mutation implicated in driving tumor growth could revolutionize the way physicians treat skin cancer.

These new drugs target a mutated form of the B-RAF oncoprotein, and cancer specialists say they represent a major improvement over existing therapies, which suffer from low response rates and fail to significantly extend survival. "We've never seen anything like this," says Lynn Schuchter, an oncologist at the University of Pennsylvania School of Medicine in Philadelphia who is involved in trials to test the new compounds and has been treating patients with metastatic melanoma, the deadliest form of the disease, for almost 25 years. "We really have tears of joy."

In January, the Swiss drug maker Roche announced preliminary phase 3 trial results showing that its experimental targeted B-RAF therapy significantly extended lifespan in people with metastatic melanoma. In fact, the study was so successful, with some participants emerging nearly cancer free, that the trial was halted prematurely, and many of the 340 subjects in the study's control arm, who previously received only standard chemotherapy, are now being given the drug. Keith Flaherty, an oncologist at Massachusetts General Hospital in Boston and principle investigator on the trial, expects federal regulators to approve the small-

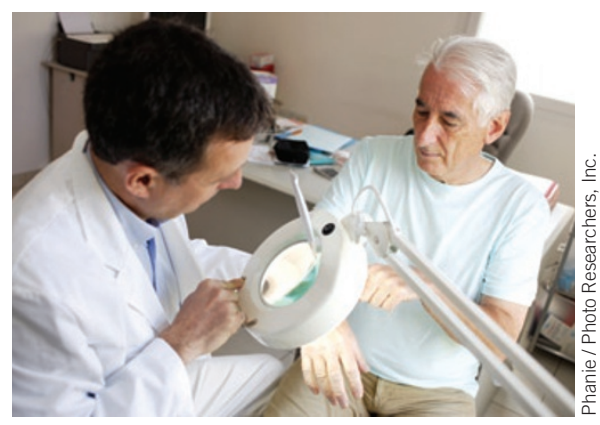

Breaking the mole: New melanoma treatments.

molecule drug, developed in partnership with Berkeley, California-based Plexxikon, later this year. "It's sort of a slam dunk," he says. Full results from the trial will be presented in June at the American Society of Clinical Oncology's annual meeting in Chicago.

The ubiquity of B-RAF mutations-first identified in 2002 and since found in many types of tumors, including lung and colon cancer cells, albeit at lower frequencies than in melanoma, where more than half of all cases of the disease express the aberrant protein-has made it an attractive target for many pharmaceutical companies. Thus, Roche is in the midst of a heated race to develop the first-in-class, mutation-specific therapy for melanoma. Also in January, GlaxoSmithKline (GSK), the British pharma giant, launched a phase 3 trial to test its own B-RAF inhibitor, and the Swiss drugmaker Novartis has another compound currently in phase 1 trials.

Despite the buzz surrounding these drugs, they aren't without side effects. Notably, some of the study subjects developed small, cancerous skin growths that had to be surgically removed, possibly as a result of nonspecific drug targeting. But, according to Keiran Smalley, a melanoma expert at the Moffitt Cancer Center in Tampa, Florida who was not involved in any of the trials, that's a small price to pay for longer survival, as people with metastatic melanoma typically survive for less than a year.

More worryingly, B-RAF inhibitors suffer from the limitation that patients nearly always develop resistance to the drugs after around six to nine months of treatment, and the tumors return or begin growing again. Researchers have just begun to sort out how this resistance occurs. Three papers published in December suggest that tumor cells can circumvent the drugs by reactivating the B-RAF pathway through other mutations or by turning on an entirely different survival pathway (Nature 468, 968-972 \& 973-977, 2010; Cancer Cell 18, 683$695,2010)$. Knowing how cancer cells evade the medications may help researchers devise drug cocktails that can prevent resistance from occurring, says Roger Lo, a dermatologist at the University of California-Los Angeles who led one of the studies and served as a subinvestigator on both the GSK and the Roche trials.

Researchers are now testing whether combining a B-RAF inhibitor with another experimental compound that blocks a separate enzyme in the same pathway will stave off resistance (see page 270). According to Richard Kefford, an oncologist at the University of Sydney in Australia and the primary investigator on a GSK combination trial, a therapy that targets two proteins at once should be "a much more durable inhibitor of cellular growth."

Cassandra Willyard

\section{Recent deal highlights hopes for cancer-killing viruses}

Can viruses be engineered to successfully tackle cancer? The biotechnology giant Amgen certainly hopes so. At the end of January, the California-based company announced that it was buying BioVex, a pioneer in developing so-called oncolytic viruses, for an impressive $\$ 1$ billion.

"Without a doubt, the Amgen deal is a validation of this field, which has often been thought of as a little bit of a backwater," says Robert Coffin, founder and chief technology officer of BioVex.

Originally spun off from University College London but now headquartered in Woburn, Massachusetts, BioVex is currently conducting phase 3 trials of OncoVEX GM-CSF, a genetically modified herpes simplex virus, for treating various cancers. It hopes to submit this vaccine for US approval at the start of 2012.
Other companies are advancing similar products. The Canadian company Oncolytics Biotech is conducting a phase 3 trial of Reolysin, a reovirus, for treating head and neck cancer. And Jennerex, headquartered in San Francisco, is conducting phase 2 trials of JX-594, a modified vaccinia virus, for treating liver cancer. Meanwhile, an engineered adenovirus for treating head and neck cancer, developed by Shanghai Sunway Biotech, was approved in China in 2005

Scientists have known for more than a century that viral infections occasionally lead to cancer remission. Subsequent studies revealed that not only can some viruses directly infect and kill cancer cells, but also this process releases antigens that prime the immune system to attack the tumor. 